\title{
Retinal detachment following extracapsular cataract extraction and posterior chamber intraocular lens implantation
}

\author{
PATRICK C $\mathrm{HO}^{12}$ AND FELIPE I TOLENTINO' \\ From the 'Eye Research Institute of Retina Foundation, Retina Associates, Massachusetts Eye and Ear \\ Infirmary, Boston, Massachusetts 02114, USA, the ${ }^{2}$ Chinese University of Hong Kong, and the ${ }^{2}$ Prince of Wales \\ Hospital, Hong Kong
}

SUMMARY Fourteen cases of primary retinal detachment after extracapsular cataract extraction and posterior chamber intraocular lens implantation were treated by scleral buckling surgery. Retinal reattachment was achieved in $100 \%$ of the cases. All eyes had a postoperative visual acuity of $6 / 30$ or better; in six eyes the visual acuity was $6 / 12$ or better. We attributed our high anatomical success rate to early detection of the retinal detachment, good visibility of the retinal breaks, lack of inflammatory reaction in the vitreous body, and preoperative absence of fixed retinal folds and preretinal membranes.

Posterior chamber intraocular lens (PC-IOL) implantation following extracapsular cataract extraction (ECCE) has recently gained much popularity among ophthalmic surgeons. ${ }^{1}$ Although retinal detachments have been mentioned as a complication of PC-IOL implantation, ${ }^{2}$ to our knowledge the clinical features of these cases have not been described. We have treated 14 cases of retinal detachment after PC-IOL implantation. Their characteristics and the course and result of their management are presented here.

\section{Materials and methods}

From 1979 to 1981 our group managed 122 consecutive cases of primary pseudophakic retinal detachment, ${ }^{3}$ of which 14 occurred after PC-IOL implantation. We were not the primary surgeons for the cataract, implant, or capsulotomy procedures, and the nature of our referral practice made it difficult to obtain details on the type of IOL and the timing and surgical technique of the capsulotomy in every case.

Each patient had a complete preoperative ophthalmic examination including gonioscopy. The fundus

Correspondence to Dr P C Ho, c/o L W Greenhill, Editorial Services, Eye Research Institute, 20 Staniford Street, Boston, Massachusetts 02114, USA (reprint requests to Library at this address). of each eye was studied by two observers, and findings were documented in large, colour-coded fundus drawings. Scleral indentation of the fundus periphery was regularly performed, as well as dynamic vitreous biomicroscopy with a three-mirror contact lens. ${ }^{4}$ We found the small-pupil binocular indirect ophthalmoscope very useful in detecting retinal breaks when the pupil was small, ${ }^{5}$ and ophthalmoscopy conducted while the patient was under anaesthesia before the start of the retinal surgery in many cases revealed retinal breaks that had not been observed during the preoperative examination.

In every case the retinal breaks were treated with cryotherapy or diathermy, and buckled with an intrascleral silicone implant with encircling band. When the ora serrata was not completely visualised for $360^{\circ}$, or when the retinal breaks could not be detected, we performed a scleral buckling that extended over the circumference of the retinal detachment and as far posteriorly to the region where the retina was visible. ${ }^{6}$ Radial buckles were used under circumferential buckles for retinal breaks posterior to the equator. Except in three cases (cases 4,7 , and 14), subretinal fluid was released slowly in a controlled fashion, and hypotony was minimised by simultaneous scleral indentation or, in two cases, by careful intravitreous air injection. Corneal endothelial damage caused by a large air bubble pushing 
Table 1 Preoperative findings

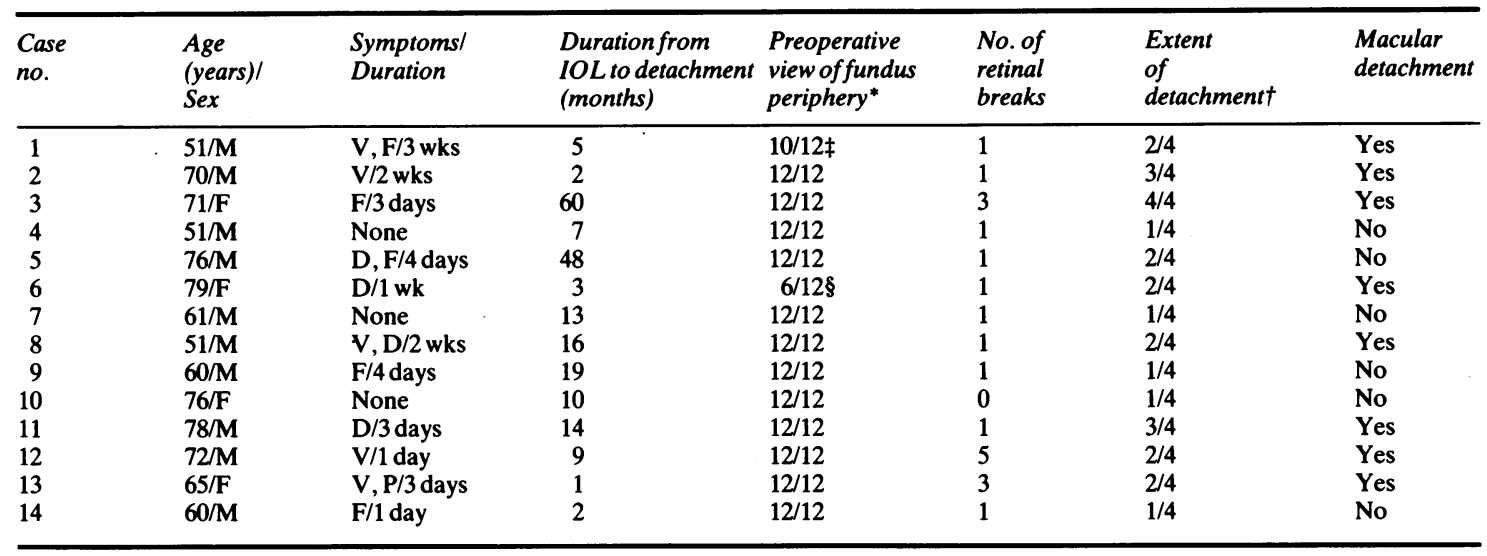

$\mathrm{V}=$ decreased visual acuity $; \mathrm{D}=$ defects in peripheral visual field $\mathrm{F}=$ floaters $\mathrm{P}=$ photopsia.

*Number of clock hours of the peripheral fundus that could be clearly observed on ophthalmoscopy.

$\dagger$ Number of quadrants of the total fundus involved. ‡Caused by vitreous pigments inferiorly. §Caused by remnants of lens cortex.

the IOL against the cornea was circumvented in one case (case 3 ) by simultaneous anterior chamber injection of an air cushion.

Postoperatively we monitored the position of the retinal breaks on the buckle and the absorption or reaccumulation of subretinal fluid with daily indirect ophthalmoscopy. Slit lamp examination was regularly performed for early detection of anterior chamber shallowing caused by postoperative choroidal effusion. ${ }^{7}$ Choroidal tap was considered only when medical therapy yielded no improvement in the corneal touch and the associated keratopathy. All successful cases of retinal reattachment were followed up for a minimum of six months.

\section{Results}

This study included 14 eyes ( 3 right, 11 left) of 14 patients (10 males, 4 females). Table 1 summarises the preoperative findings. Three patients did not present with any symptoms when the peripheral quadrantic retinal detachment was discovered on routine ocular examination. In eight cases the retina became detached within a year after the cataract surgery and IOL implantation, with four cases occurring within the first three months. A history of intraoperative vitreous loss was not elicited from any of the referring cataract surgeons. However, vitreous gel was observed preoperatively to herniate through the posterior capsulotomy and around the PC-IOL into the anterior chamber in cases 3 and 12 . Posterior capsulotomy was detected in all 14 cases. On ophthalmoscopy there was no difficulty in viewing the fundus periphery except in two cases; one had heavily pigmented opacities in the inferior vitreous base, and the other had significant peripheral cortical remnants from incomplete extracapsular lens extraction. In both these eyes the inability to observe the ora serrata for its entire $360^{\circ}$ did not compromise the success of locating the retinal breaks. In 11 of the 14 cases the detachment involved one or two quadrants of the fundus, and in six cases the macula was still attached at the time the retinal detachment was detected. In two of the eight eyes in which the macula was detached preoperatively (cases 1 and 8 ), the preoperative visual acuities were not profoundly depressed before the diagnosis was made. We took particular notice of the clarity of the vitreous and of the absence of preretinal membranes, full-thickness retinal folds, and star folds.

Intraoperatively, in addition to scleral buckling, intravitreous air injection was performed in two eyes (cases 3 and 13) to tamponade the retinal breaks, which were complicated by the fish-mouth phenomenon. In case 13 the retinal operation undertaken one month after the cataract operation was complicated by corneal wound leakage, which was repaired with additional corneal sutures. Retina reattachment was achieved in all 14 eyes. During the follow-up period retinal detachment did not recur, and we did not observe any signs of vitreoretinal proliferation. ${ }^{8}$ Additional operations were performed, however, on three eyes. In case 8 the surgeon elected to reinforce the chorioretinal adhesions around the retinal break with photocoagulation in the immediate postoperative period. Choroidal effusion was observed in three eyes after the primary scleral buckling procedure. In two eyes the effusion 
Table 2 Operative findings and results

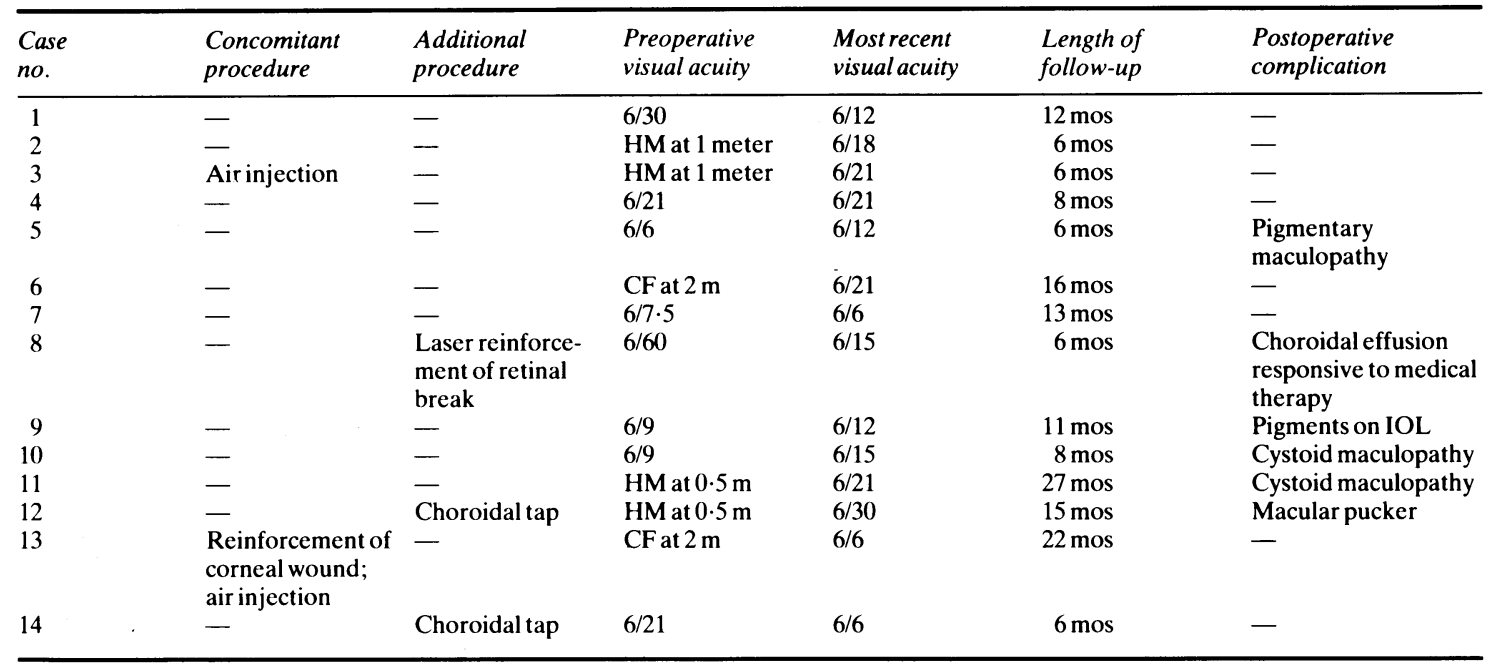

$\mathrm{HM}=$ hand movements $; \mathrm{CF}=$ counting fingers .

persisted despite medical therapy, and was finally relieved by uneventful choroidal tap. Table 2 lists the operative procedures, findings, and results.

At the most recent follow-up visit 10 eyes had improved visual acuity of one line or more on the Snellen chart. In one eye there was no change, and in three eyes the postoperative visual acuities were worse than the preoperative ones. In these three cases the contributing causes were pigment clumps in the macula, persistent cystoid maculopathy, and pigment dispersion on the surfaces of the IOL. None of our cases had visual acuity worse than $6 / 30$ postoperatively, and visual acuity of $6 / 12$ or better was achieved in six cases $(43 \%)$.

\section{Discussion}

One of the proposed theoretical advantages of ECCE and PC-IOL over conventional methods of cataract extraction has been the preservation of vitreous stability in the posterior segment, minimising the occurrence of vitreoretinal traction, cystoid macular oedema, and retinal detachment. ${ }^{9}$ Although ECCE with PC-IOL has gained much popularity among ophthalmic surgeons, it is still a relatively new procedure, and the eyes that have undergone this operation have not been followed up long enough to permit a reliable determination of the incidence of postoperative retinal detachment. The nature of our retinal referral practice does not allow us to draw from our data any conclusion pertaining to this incidence. Although the number of cases in this study is relatively small, certain trends are apparent.
Retinal reattachment was achieved in all our cases. This reattachment rate of $100 \%$ could be attributed to a number of factors. In many of our cases the retinal detachment was diagnosed and repaired before it involved the entire fundus. Perhaps patients with PC-IOLs were more aware of changes in their peripheral visual fields and more likely to seek medical attention soon after the onset of symptoms. In addition to making an earlier diagnosis of retinal detachment in most of our cases we also found no difficulty in visualising the retinal breaks. In only one eye were the peripheral cortical remnants significant enough to obstruct the view of 6 clock hours of the ora serrata, but this did not prevent locating the retinal break contributing to the detachment. The recent boom in ophthalmic technology in improving microsurgical instruments, the refined microsurgical techniques, and the increasing experience of ocular surgeons in mastering the extracapsular procedure all lead to thorough removal of peripheral lens cortex in most of these ECCE cases.

The success rate of any clinical study concerning the surgical management of retinal detachment depends on the number of cases with proliferative vitreoretinopathy ${ }^{38}$ included in the study population. In our cases we were impressed by the lack of vitreous reactions both preoperatively and postoperatively. In no instance did we observe any full-thickness fixed retinal folds, preretinal membranes, or haziness of the vitreous gel. It is likely that the conditions at the time of the cataract extraction that would permit the safe implantation of a PC-IOL would also produce the least disturbance in the vitreous body during the 
operation and after it. Perhaps the short duration of the retinal detachment before it was diagnosed and repaired (Table 1) also contributed to the absence of preretinal membranes and therefore indirectly to the results obtained.

Thus, in this study, although we could not substantiate the claim that ECCE and PC-IOL minimise the incidence of postoperative retinal detachment, we have shown that, at least in our cases, when retinal detachment occurred in eyes following ECCE and PC-IOL, the prognosis of retinal reattachment with surgical repair could be excellent.

Records from Drs I D Okamura, J W McMeel, H M Freeman, and R C Pruett were also included in this study.

This work was supported in part by the Massachusetts Lions Eye Research Fund, Inc.

\section{References}

1 Stark WJ, Leske MC, Worthen DM, Murray GC. Trends in cataract surgery and intraocular lenses in the United States. $\mathbf{A m ~ J}$ Ophthalmol 1983; 96: 304-10.

2 Fagadau WR, Maumenee AE, Stark WJ Jr, Datiles M. Posterior chamber intraocular lenses at the Wilmer Institute: a comparative analysis of complications and visual results. $\mathrm{Br} J$ Ophthalmol 1984; 68: $13-8$.

3 Ho PC, Tolentino FI. Pseudophakic retinal detachment: surgical success rate with various types of IOLs. Ophthalmology (Rochester) 1984; 91: 847-52.

4 Tolentino FI, Schepens CL, Freeman HM. Vitreoretinal disorders: diagnosis and management. Philadelphia: Saunders, 1976: 71-108.

5 Hovland KR, Elzeneiny IH, Schepens CL. Clinical evaluation of the small-pupil binocular indirect ophthalmoscope. Arch Ophthalmol 1969; 82: 466-74.

6 Freeman HM, Dobbie JG, Friedman MW, et al. Pseudophakic retinal detachment. Mod Probl Ophthalmol 1979; 20: 345-53.

7 Freeman HM. Discussion of: Pseudophakic flat anterior chamber following retinal detachment repair, by Taylor BC, Winslow RL. Ophthalmology (Rochester) 1981; 88: 940-1.

8 The Retina Society Terminology Committee. The classification of retinal detachment with proliferative vitreoretinopathy. Ophthalmology (Rochester) 1983; 90: 121-5.

9 Ho PC, Tolentino FI. The role of vitreous in aphakic cystoid macular edema: a review. Am Intraocular Implant Soc J 1982; 8: 258-64. 